\title{
Early Annihilation and Diffuse Backgrounds in Models of Weakly Interacting Massive Particles in Which the Cross Section for Pair Annihilation Is Enhanced by $1 / v$
}

\author{
Marc Kamionkowski ${ }^{1}$ and Stefano Profumo ${ }^{2}$ \\ ${ }^{1}$ California Institute of Technology, Mail Code 130-33, Pasadena, California 91125, USA \\ ${ }^{2}$ Santa Cruz Institute for Particle Physics and Department of Physics, University of California, Santa Cruz, California 95064, USA
}

(Received 20 October 2008; published 29 December 2008)

\begin{abstract}
Recent studies have considered modifications to the standard weakly interacting massive particle scenario in which the pair annihilation cross section (times relative velocity $v$ ) is enhanced by a factor $1 / v \sim 10^{-3}$ in the Galaxy, enough to explain several puzzling Galactic radiation signals. We show that in these scenarios a burst of weakly interacting massive particle annihilation occurs in the first collapsed dark-matter halos. We show that severe constraints to the annihilation cross section derive from measurements of the diffuse extragalactic radiation and from ionization and heating of the intergalactic medium.
\end{abstract}

DOI: 10.1103/PhysRevLett.101.261301

In the standard weakly interacting massive particle (WIMP) scenario [1-3], dark matter is composed of particles that have electroweak interactions with ordinary matter. Such particles cease to annihilate to standard-model particles in the primordial plasma to produce a cosmological relic density that is generically in the ballpark of that required to account for the dark matter. The most widely studied WIMPs are the neutralino, the lightest supersymmetric particle in several supersymmetric extensions to the standard model [1], and, more recently, the lightest KaluzaKlein particle in models with universal extra dimensions [4].

Typical values for the WIMP mass are $m_{\chi} \sim 10 \mathrm{GeV}-\mathrm{a}$ few $\mathrm{TeV}$, and freeze-out of annihilations (chemical decoupling) occurs at temperatures $T_{f} \sim m_{\chi} / 20$. After freezeout, the WIMP temperature remains fixed to the temperature of the primordial plasma via frequent elastic scattering from standard-model particles. When the temperature drops below the kinetic-decoupling temperature $T_{\mathrm{kd}}$, which generally falls in WIMP models in the range $T_{\mathrm{kd}} \sim$ $10 \mathrm{MeV}-\mathrm{a}$ few $\mathrm{GeV}$ [5,6], WIMPs kinetically decouple from the plasma, and their temperature subsequently decays with the scale factor $R$ as $R^{-2}$, rather than $R^{-1}$. Afterwards, WIMPs are effectively collisionless; they behave in the subsequent universe like the cold dark matter required to account for detailed measurements of the cosmic microwave background (CMB) and large-scale structure.

However, a fraction of WIMPs could annihilate, once galactic halos form later in the universe, to produce cosmic gamma rays or cosmic-ray antiprotons or positrons $[1,3]$. In the standard WIMP scenario, the annihilation cross section (times relative velocity $v$ ) can be cast as $(\sigma v)_{\text {ann }} \simeq$ $a+b(v / c)^{2}+\cdots$. Roughly speaking, this cross section must evaluate at $v \sim c / 2$ to $(\sigma v)_{\mathrm{ann}} \simeq 3 \times 10^{-26} \mathrm{~cm}^{3} \mathrm{~s}^{-1}$ to obtain the correct relic density, while annihilation in the Galactic halo occurs at $v \sim 10^{-3}$, where $(\sigma v)_{\mathrm{ann}} \simeq a \lesssim$ $3 \times 10^{-26} \mathrm{~cm}^{3} \mathrm{~s}^{-1}$. The fluxes of cosmic rays from WIMP
PACS numbers: 95.35.+d, 98.62.Gq, 98.70.Vc

annihilation are thus generically expected to be very small; detection would require some mechanism to boost the annihilation rate typically by a few orders of magnitude $[3,7]$.

In recent years, there have been several unidentified Galactic radiation backgrounds. These include a diffuse synchrotron haze around the Galactic center in the Wilkinson Microwave Anisotropy Probe (WMAP) data [8], a 511-keV gamma-ray excess from the Galactic center [9], an excess high-energy gamma-ray background from the Galactic center [10], and, most recently, a reported detection of an upturn in the cosmic-ray positron fraction at high energies [11]. Efforts have been made to explain these Galactic radiation backgrounds in terms of WIMP annihilation, but some mechanism to boost the annihilation rate, relative to the natural expectation, must be introduced.

One way to boost the annihilation rate is to alter the underlying particle theory so that the WIMP annihilation cross section (times relative velocity) goes as $1 / v$ (rather than to a constant, as in the standard scenario) as $v \rightarrow 0$. Such a cross section is consistent with $s$-wave unitarity [12] as long as $(\sigma v)_{\text {ann }} \leq 4 \pi /\left(m_{\chi}^{2} v\right)$ as $v \rightarrow 0$. Even larger annihilation cross sections are conceivable if higher partial waves contribute. Specific mechanisms for providing a low-velocity enhancement include the Sommerfeld enhancement and/or the formation of bound particleantiparticle states in which the particles then annihilate [13] although the functional dependence is not always precisely $1 / v$, an issue we elaborate on further below.

Here we parametrize the $1 / v$ enhancement by writing $(\sigma v)_{\text {ann }}=3 \times 10^{-26} \sigma_{26}(c / v) \mathrm{cm}^{3} \mathrm{~s}^{-1}$, and we refer to these models as " $1 / v$ WIMP models" or just " $1 / v$ models." Since the cross section at freeze-out required to obtain the relic abundance is roughly $3 \times 10^{-26} \mathrm{~cm}^{3} \mathrm{~s}^{-1}$, we infer that the parameter $\sigma_{26}$ must be $\sigma_{26} \lesssim 1$ or else the WIMP abundance will be too small. The equality is obtained if there are no other contributions to the annihilation cross section; $\sigma_{26}$ could be smaller if there are other contributions (e.g., $a+b v^{2}$ ) to the annihilation cross section. 
We make the relic-abundance constraint to $\sigma_{26}$ more precise by including the $1 / v$ dependence of $(\sigma v)_{\text {ann }}$ in the solution to the Boltzmann equation [14]. Doing so, we estimate the relic abundance of $1 / v$ models as $\left(\Omega_{\chi} h^{2} / 0.1\right) \simeq(1 / 2) \sqrt{\pi / x_{f}} \sigma_{26}^{-1}$, with $x_{f}=m_{\chi} / T_{f} \sim 20$. This implies $\sigma_{26} \lesssim 0.2$ to explain the observed darkmatter density, the inequality occurring, again, if there are other contributions to the annihilation cross section.

We now describe the bounds to this scenario that arise from upper limits to the diffuse extragalactic gamma-ray background $(\gamma \mathrm{RB})$ and from CMB constraints to the ionization history. After the universe becomes matter dominated, perturbations in the cold-dark-matter density grow. The smallest structures undergo gravitational collapse first, and then more massive structures collapse later. The kinetic coupling of WIMPs at temperatures $T_{f} \gtrsim T \gtrsim T_{\mathrm{kd}}$ erases primordial structure on mass scales smaller than $M_{c} \simeq 33\left(T_{\mathrm{kd}} / 10 \mathrm{MeV}\right)^{-3} M_{\oplus}$ [15]. The first gravitationally bound structures in the hierarchy therefore have masses $M_{c}$. The first objects collapse at a redshift that can be approximated over the range $10^{-6} M_{\oplus} \lesssim M_{c} \lesssim 100 M_{\oplus}$ relevant for WIMPs [5] by $z_{c}=140-\log _{10}\left(M_{c} / M_{\oplus}\right)$. (This approximation is obtained with a scalar spectral index $n_{s}=1$. It will change slightly for $n_{s} \simeq 0.95$. We include the $z_{c}$ and $M_{c}$ dependences separately in subsequent expressions so that a different $z_{c}-M_{c}$ relation can be used.) These protohalos collapse to a virial density $\rho$ that is $\approx 178$ times the mean cosmological density at the collapse redshift $z_{c}$. The velocity dispersion in the protohalos can be approximated by $v \sim R / t$, where $t \sim$ $(G \rho)^{-1 / 2}$ is the dynamical time and $R \sim(M / \rho)^{1 / 3}$ is the size of the halos. Thus, $v \sim M^{1 / 3} G^{1 / 2} \rho^{1 / 6}$. Numerically, the first halos of mass $M_{c}$ that collapse at redshift $z_{c}$ will have velocity dispersions

$$
(v / c) \sim 6.0 \times 10^{-9}\left(M_{c} / M_{\oplus}\right)^{1 / 3}\left(z_{c} / 200\right)^{1 / 2} .
$$

The rate at which WIMPs annihilate in this first generation of halos is $\Gamma=n_{\chi}(\sigma v)_{\text {ann }}$, which evaluates to

$$
\Gamma=2.2 \times 10^{-17}\left(\frac{M_{c}}{M_{\oplus}}\right)^{-1 / 3} \sigma_{26} B_{2.6}\left(\frac{z_{c}}{200}\right)^{5 / 2}\left(\frac{m_{\chi}}{\mathrm{TeV}}\right)^{-1} \mathrm{~s}^{-1} .
$$

Here we have included a boost factor [16]

$$
B \equiv \frac{\int \rho^{2} d V}{V \rho_{v}^{2}}=\frac{c_{v}^{3} g\left(c_{v}\right)}{3\left[f\left(c_{v}\right)\right]^{2}}
$$

for a Navarro-Frenk-White (NFW) density profile [17], where $c_{v}$ is the concentration parameter, $g\left(c_{v}\right)=(1 / 3) \times$ $\left[1-\left(1+c_{v}\right)^{-3}\right]$, and $f\left(c_{v}\right)=\ln c_{v}-c_{v} /\left(1+c_{v}\right)$. This boost factor takes into account the increase in the annihilation rate due to the fact that the dark matter is distributed in these first halos with an NFW density profile $\rho(r)$, rather than uniformly distributed with density $\rho$. The boost factor varies from $B=2.6$ for $c_{v}=1$ to $B=50$ for $c_{v}=10$. To be conservative, we adopt $B=2.6$ but include the $B$ dependence through the parameter $B_{2.6} \equiv B / 2.6$ in all subsequent expressions. Strictly speaking, one should also integrate $(\sigma v)_{\text {ann }}$, which now depends on $v$, over the velocity distribution in the halo. However, since the mean inverse velocity $\left\langle v^{-1}\right\rangle$ will not be too different for different velocity distributions with the same mean velocity $\langle v\rangle$, this added uncertainty should give no more than an order-unity correction to the annihilation rate [Eq. (2)]. We thus fold this added theoretical uncertainty into the parameter $B_{2.6}$.

The first generation of halos survive roughly a Hubble time before they merge into slightly higher-mass (and lower-density) halos. The age of the universe at redshifts $z \gg 1$ is $t \simeq 2 \times 10^{14}\left(z_{c} / 200\right)^{-3 / 2} \mathrm{~s}$. We thus infer that the fraction of dark-matter particles that annihilate in the first generation of halos is

$$
f \simeq \Gamma t \simeq 4.4 \times 10^{-3}\left(\frac{M_{c}}{M_{\oplus}}\right)^{-1 / 3} \sigma_{26} B_{2.6}\left(\frac{z_{c}}{200}\right)\left(\frac{m_{\chi}}{\mathrm{TeV}}\right)^{-1} .
$$

To begin, we note that a relatively weak bound to the parameter space can be obtained by noting that measurements of the CMB power spectrum constrain the matter density at recombination to be within $10 \%$ of its value today. We thus infer that $f \leqq 0.1$, independent of any knowledge of the annihilation products.

However, if $1 / v$ models are introduced to explain Galactic radiation backgrounds, they require annihilation into $e^{+} e^{-}$pairs and/or gamma rays. As shown in Fig. 2 of Ref. [18], photons injected with energies $E_{i}$ at redshifts $z_{c} \sim 100-200$ in the range $100 \mathrm{keV} \lesssim E_{i} \lesssim 300 \mathrm{GeV}$ propagate freely through the universe, with energies that decrease with redshift as they propagate. They thus appear to us as a diffuse extragalactic background of gamma rays with energy $E_{\gamma}=E_{i} / z_{c}$. Photons injected at $z \sim 100$ with energies $E_{i} \lesssim 100 \mathrm{keV}$ propagate at first through the universe but then get absorbed at lower redshift by the intergalactic medium (IGM). Photons injected with energies $\gtrsim 300 \mathrm{GeV}$ get absorbed immediately by the IGM.

Electron-positron pairs injected into the universe at redshifts $z \sim 100-200$ with energy $E_{e}$ very rapidly inverseCompton scatter CMB photons resulting in a gamma ray of energy $E_{i} \sim\left(E_{e} / m_{e}\right)^{2} T_{\mathrm{CMB}}$, where $T_{\mathrm{CMB}} \sim 10^{-2} \mathrm{eV}$ is the characteristic CMB-photon energy at these redshifts. Thus, electron-positron pairs injected with energies in the range $\mathrm{GeV} \lesssim E_{e} \lesssim 2 \mathrm{TeV}$ produce photons in the energy range $100 \mathrm{keV} \lesssim E_{i} \lesssim 300 \mathrm{GeV}$ of the transparency window; these gamma rays then appear to us as a diffuse background. Electrons injected at $z \sim 100-200$ with energies $E_{e} \lesssim \mathrm{GeV}$ get absorbed by the IGM at low redshifts, and those at $E_{e} \gtrsim 2 \mathrm{TeV}$ are absorbed by the IGM immediately at high redshift.

If the photons (or electron-induced photons) are not absorbed by the IGM, then the energy density in photons today from WIMP annihilation in the first halos is simply $\rho_{\gamma}=f \rho_{\chi}^{0} / z_{c}$, i.e., the fraction of the WIMP energy density that gets converted to radiation through annihilation, 
scaled by the redshift of the photons. This evaluates numerically to

$$
\rho_{\gamma}=2.64 \times 10^{-11}\left(\frac{M_{c}}{M_{\oplus}}\right)^{-1 / 3} \sigma_{26} B_{2.6}\left(\frac{m_{\chi}}{\mathrm{TeV}}\right)^{-1} \mathrm{GeV} \mathrm{cm}^{-3}
$$

(Ref. [19] also considered the $\gamma$ RB from WIMP annihilation in the first objects, although they did not consider the $1 / v$ enhancement). We now compare this with the upper limit $\rho_{\gamma} \lesssim 5.7 \times 10^{-16}\left(E_{\gamma} / \mathrm{GeV}\right)^{-0.1} \mathrm{GeV} \mathrm{cm}^{-3}[18,20]$ to obtain the constraint

$$
\sigma_{26} \lesssim 2.2 \times 10^{-5} B_{2.6}^{-1}\left(\frac{M_{c}}{M_{\oplus}}\right)^{1 / 3}\left(\frac{E_{\gamma}}{\mathrm{GeV}}\right)^{-0.1}\left(\frac{m_{\chi}}{\mathrm{TeV}}\right) .
$$

This upper bound is derived for $30 \mathrm{MeV} \lesssim E_{\gamma} \lesssim$ $100 \mathrm{GeV}$. It is a fit to the extragalactic $\gamma \mathrm{RB}$, much of which comes from unresolved astrophysical sources; it is thus a conservative upper limit. Moreover, the recently launched Fermi-GLAST telescope should soon significantly improve the upper limit to this $\gamma \mathrm{RB}$. Equation (6) is a conservative upper limit all the way down to energies $\sim \mathrm{keV}$. The true upper limits from $\mathrm{x}$-ray-background and $\gamma \mathrm{RB}$ measurements in the energy range $10 \mathrm{keV} \lesssim E_{\gamma} \lesssim$ $30 \mathrm{MeV}$ are more stringent. Notice that the limit in Eq. (6) does not depend on the collapse redshift $z_{c}$.

There will continue to be WIMP annihilation during later, more massive, stages in the structure-formation hierarchy. These will produce higher-energy photons (since they get redshifted less), but the energy density in these higher-energy photons will be far smaller. This can be seen from Eq. (4) by noting that the factor $M_{c}$ that appears therein will be replaced by $M_{*}(z)$, the characteristic halo mass at redshift $z$ (interestingly enough, all other redshift dependence in that equation cancels). The mass scale $M_{*}(z)$ evolves very rapidly with $z$; e.g., it goes from $10^{-12} M_{\odot}$ to $10^{-4} M_{\odot}$ from $z \simeq 200$ to $z \simeq 100$, and then all the way to $\sim 10^{14} M_{\odot}$ at $z=0$. The constraint to the model from the diffuse-background flux is thus strongest from the earliest halos.

Let us now consider what happens if the photon (or electron-induced photon) falls outside the transparency window, i.e., if it is injected with energy $E_{i} \lesssim 100 \mathrm{keV}$ or $E_{i} \gtrsim 300 \mathrm{GeV}$. In both cases, the photons are absorbed by the IGM before they can reach us, and so there is no constraint from diffuse backgrounds. In both cases, though, very stringent constraints arise from measurements of CMB temperature and polarization [18,21,22]. These photons ionize and heat the IGM. The reionized electrons scatter $\mathrm{CMB}$ photons, thus altering the observed CMB temperature or polarization power spectra. Reference [22] carried out detailed fits to WMAP3 and large-scale-structure data to constrain the heating or ionization of the IGM. We infer from Fig. 2 in Ref. [22] that no more than a fraction $f \lesssim 10^{-9}$ of the rest-mass energy of the dark matter could have been injected into the IGM at a time $\sim 10^{15} \mathrm{sec}$ after the big bang. The magnitude of the upper limit can be understood simply: Dark matter outweighs baryons by a factor of 6 , and it requires a fraction $(10 \mathrm{eV} / \mathrm{GeV}) \sim 10^{-8}$ of the rest-mass energy of each atom to ionize it.

Thus, if dark matter annihilates to photons with energies $E_{i} \gtrsim 300 \mathrm{GeV}$ or to electron-positron pairs with energies $E_{e} \gtrsim 2 \mathrm{TeV}$, then we require

$$
\sigma_{26} \lesssim 2.3 \times 10^{-7}\left(\frac{M_{c}}{M_{\oplus}}\right)^{1 / 3} B_{2.6}^{-1}\left(\frac{z_{c}}{200}\right)^{-1}\left(\frac{m_{\chi}}{\mathrm{TeV}}\right) .
$$

The result for photons injected with energies $E_{\gamma} \lesssim$ $100 \mathrm{keV}$ (or electron-positron pairs with $E_{e} \lesssim \mathrm{GeV}$ ) is similar but weakened possibly by the redshift of the photon energy that occurs between the time it was injected and the time it was absorbed. The detailed suppression depends on the injected energy and redshift. However, in no case is the suppression stronger than a factor $z_{c}^{-1}$. We thus conclude that the bound will be no more than 2 orders of magnitude weaker than that quoted in Eq. (7).

In summary, there will be a burst in $1 / v$ WIMP models of annihilation in dark matter that form at redshifts $z \sim$ 100-200. There is a weak, albeit final-state-independent, CMB bound that amounts to demanding that no more than $\sim 10 \%$ of the dark matter annihilates after recombination. If the WIMP annihilates to photons in the energy range $100 \mathrm{keV} \lesssim E_{i} \lesssim 300 \mathrm{GeV}$ or to electrons in the energy range $\mathrm{GeV} \lesssim E_{e} \lesssim 2 \mathrm{TeV}$, then there are constraints, summarized in Eq. (6), to the cross section for annihilation to $e^{+} e^{-}$pairs and photons. If the photons or electrons are injected outside the transparency window, then there is a bound, quoted in Eq. (7), that comes from CMB constraints to ionization of the IGM.

To clarify, the bounds to $\sigma_{26}$ in Eqs. (6) and (7) are to the cross section for annihilation to photons and $e^{+} e^{-}$pairs, not to the total annihilation cross section. A large hadronic final-state branching ratio is, however, greatly constrained by antiproton data [23]. Note also that (i) $B_{2.6}$ is likely larger than unity, that (ii) $z_{c}$ is generally larger than 100 for typical WIMP models, and that (iii) $M_{c}$ is generally smaller than $M_{\oplus}$. The numerical values in Eqs. (6) and (7) will, for typical WIMP setups, generally be smaller. On the other hand, the numerical value in Eq. (6) may be larger for smaller $E_{i}$.

For the nominal WIMP values that we have chosen, the bounds are violated by 4-6 orders of magnitude, depending on the gamma-ray energy, and the constraints are still $\sigma_{26} \ll 1$ for almost any combination of the parameters $E_{\gamma}, M_{c}$, and $z_{c}$ consistent with typical WIMP values. Specifically, to obtain the enhancements $\mathcal{O}\left(10^{2}\right)$ needed by some dark-matter interpretations of recent cosmic-ray measurements with a Galactic $c / v \sim 10^{3}$, one requires $\sigma_{26} \geqslant 0.1$, inconsistent with the limits derived here. Thus, something else must be invoked in a $1 / v$ model to evade the $\gamma \mathrm{RB}$ and/or ionization-history constraint.

First consider the Sommerfeld enhancement [13]. The $1 / v$ scaling is valid only for $(v / c) \gtrsim\left(m_{\phi} / m_{\chi}\right)$, where $m_{\phi}$ 
is the mass of a light exchanged particle. At smaller velocities, the $1 / v$ enhancement saturates at $m_{\chi} / m_{\phi}$. Our bounds can therefore be written for this model, roughly speaking, by including a factor $\max \left[1,(c / v)\left(m_{\phi} / m_{\chi}\right)\right]$, with $v / c$ evaluated from Eq. (1), on the right-hand sides of our upper limits [Eqs. (6) and (7)]. Thus, for example, for our canonical values $\left[m_{\chi}=\mathrm{TeV}, M_{c}=M_{\oplus}, z_{c}=200\right.$, and $B_{2.6}=1$ ], our limits are unaltered for $m_{\phi} \lesssim 6 \mathrm{keV}$. For larger $m_{\phi}$, they are reduced accordingly. For example, the CMB bound [Eq. (7)] is weakened to $\sigma_{26} \lesssim 1$ (for our canonical values) for $m_{\phi} \gtrsim 26 \mathrm{GeV}$.

We mention two ways to evade the bound, even if the $1 / v$ scaling extends to $v=0$, one astrophysical and one of particle-physics origin: The first possibility is that the first microhalos that form at redshift $z \sim 100-200$ remain largely intact through subsequent stages in the merger hierarchy. The Galactic halo then consists of a huge number of distinct $\lesssim M_{\oplus}$ microhalos, each of which has a density $\sim 10^{6}$ times the mean halo density and velocity dispersion $\sim 10^{-6}$ times the Milky Way velocity dispersion [24]. A far larger $\left(\sim 10^{12}\right)$ boost factor would then be predicted for annihilation in the Milky Way halo than the value $v^{-1} \sim 10^{3}$ obtained by assuming a smooth halo. This would then allow a far smaller value $\sigma_{26}$ to produce the annihilation required to explain some of the unidentified Galactic radiation backgrounds. Smaller values of $\sigma_{26}$ would, however, require the $1 / v$-independent terms in the annihilation cross section to obtain the correct relic abundance. This solution would require the survival of a significant fraction of early microhalos; the precise survival probability remains debated in the literature.

A second possibility is to design a model in which the cross section for a WIMP to elastically scatter from photons and neutrinos is also enhanced. This would allow the WIMP to remain in kinetic equilibrium until lower temperatures, resulting in a larger $M_{c}$. This would then imply a suppression of the limits from the $\gamma \mathrm{RB}$ or IGM accordingly. In the extreme scenario, dark-matter halos would not form until $z_{v} \sim 10$, when the first halos and the stars they house would have had to form to reionize the universe. We leave the construction of such models for future work.

We acknowledge useful comments from N. ArkaniHamed, J. Feng, D. Finkbeiner, R. Gilmore, M. Pospelov, M. Ritz, and N. Weiner. This work was supported at Caltech by DoE No. DE-FG03-92-ER40701 and the Gordon and Betty Moore Foundation.

[1] G. Jungman, M. Kamionkowski, and K. Griest, Phys. Rep. 267, 195 (1996).

[2] L. Bergstrom, Rep. Prog. Phys. 63, 793 (2000).

[3] G. Bertone, D. Hooper, and J. Silk, Phys. Rep. 405, 279 (2005).

[4] H. C. Cheng, J. L. Feng, and K. T. Matchev, Phys. Rev. Lett. 89, 211301 (2002); G. Servant and T. M.P. Tait,
Nucl. Phys. B650, 391 (2003); for a review, see D. Hooper and S. Profumo, Phys. Rep. 453, 29 (2007).

[5] S. Profumo, K. Sigurdson, and M. Kamionkowski, Phys. Rev. Lett. 97, 031301 (2006).

[6] T. Bringmann and S. Hofmann, J. Cosmol. Astropart. Phys. 04 (2007) 016.

[7] E. A. Baltz et al., J. Cosmol. Astropart. Phys. 07 (2008) 013.

[8] D. P. Finkbeiner, Astrophys. J. 614, 186 (2004); G. Dobler and D. P. Finkbeiner, Astrophys. J. 680, 1222 (2008); D. Hooper, D. P. Finkbeiner, and G. Dobler, Phys. Rev. D 76, 083012 (2007).

[9] P. Jean et al., Astron. Astrophys. 407, L55 (2003); J. Knodlseder et al., Astron. Astrophys. 411, L457 (2003); G. Weidenspointner et al., arXiv:astro-ph/0406178.

[10] A. W. Strong et al., Astron. Astrophys. 444, 495 (2005); D. J. Thompson, D. L. Bertsch, and R. H. O'Neal, arXiv: astro-ph/0412376.

[11] O. Adriani et al., arXiv:0810.4995.

[12] K. Griest and M. Kamionkowski, Phys. Rev. Lett. 64, 615 (1990).

[13] J. Hisano et al., Phys. Rev. D 71, 063528 (2005); J. MarchRussell et al., J. High Energy Phys. 07 (2008) 058; N. Arkani-Hamed et al., arXiv:0810.0713; M. Pospelov and A. Ritz, arXiv:0810.1502; J. L. Feng and J. Kumar, Phys. Rev. Lett. 101, 231301 (2008); J. L. Feng, H. Tu, and H. B. Yu, J. Cosmol. Astropart. Phys. 10 (2008) 043.

[14] R. J. Scherrer and M. S. Turner, Phys. Rev. D 33, 1585 (1986); 34, 3263(E) (1986).

[15] A. Loeb and M. Zaldarriaga, Phys. Rev. D 71, 103520 (2005); E. Bertschinger, Phys. Rev. D 74, 063509 (2006).

[16] M. Kamionkowski and S. M. Koushiappas, Phys. Rev. D 77, 103509 (2008).

[17] J. F. Navarro, C. S. Frenk, and S. D. M. White, Astrophys. J. 490, 493 (1997).

[18] X.L. Chen and M. Kamionkowski, Phys. Rev. D 70, 043502 (2004).

[19] T. Oda, T. Totani, and M. Nagashima, Astrophys. J. 633, L65 (2005).

[20] P. Sreekumar et al. (EGRET Collaboration), Astrophys. J. 494, 523 (1998).

[21] E. Pierpaoli, Phys. Rev. Lett. 92, 031301 (2004); S. Kasuya and M. Kawasaki, Phys. Rev. D 70, 103519 (2004); L. Zhang, X. L. Chen, Y. A. Lei, and Z. G. Si, Phys. Rev. D 74, 103519 (2006); A. G. Doroshkevich et al., Astrophys. J. 586, 709 (2003); R. Bean, A. Melchiorri, and J. Silk, Phys. Rev. D 68, 083501 (2003); S. H. Hansen and Z. Haiman, Astrophys. J. 600, 26 (2004); S. Kasuya, M. Kawasaki, and N. Sugiyama, Phys. Rev. D 69, 023512 (2004); N. Padmanabhan and D. P. Finkbeiner, Phys. Rev. D 72, 023508 (2005); M. Mapelli, A. Ferrara, and E. Pierpaoli, Mon. Not. R. Astron. Soc. 369, 1719 (2006); R. Bean, A. Melchiorri, and J. Silk, Phys. Rev. D 75, 063505 (2007); S. Kasuya and M. Kawasaki, J. Cosmol. Astropart. Phys. 02 (2007) 010.

[22] L. Zhang et al., Phys. Rev. D 76, 061301 (2007).

[23] L. Bergstrom et al., J. Cosmol. Astropart. Phys. 05 (2006) 006.

[24] J. Diemand et al., Nature (London) 454, 735 (2008). 